\title{
A GENERALIZATION OF THE EICHLER TRACE FORMULA FOR MORPHISMS BETWEEN RIEMANN SURFACES
}

\author{
Masaharu Tanabe \\ Tokyo Institute of Technology, Department of Mathematics \\ Ohokayama, Meguro, Tokyo, 152-8551, Japan; tanabe.m.ac@m.titech.ac.jp
}

\begin{abstract}
Let $T$ be an automorphism of a closed Riemann surface. The Eichler trace formula asserts that the trace of the pull-back action of $T$ on the space of holomorphic 1-forms can be evaluated in terms of the local behavior of $T$ around the fixed points. In this paper, we will generalize this formula for morphisms between closed Riemann surfaces of possibly different genera.
\end{abstract}

\section{Introduction}

Throughout this paper, all of the Riemann surfaces are closed and of genera $\geq 1$. We recall the Eichler trace formula. Let $T$ be an automorphism of order $q$ of a Riemann surface $X$. Suppose that there are $t$ fixed points $p_{1}, p_{2}, \cdots, p_{t}$. We put $\zeta=e^{2 \pi i / q}$. Choosing a coordinated neighbourhood around $p_{j}$ properly, we may write

$$
T: z \rightarrow \zeta^{k_{j}} z
$$

for some $k_{j}, 1 \leq k_{j} \leq q-1$ in the neighbourhood of $p_{j}$. $T$ induces the pull-back action on the space of holomorphic 1 -forms on $X$. Let $\chi$ denote the trace of a matrix representation of the action on the space of holomorphic 1-forms. Then the Eichler trace formula is

$$
\chi=1-\sum_{j=1}^{t} \frac{\zeta^{k_{j}}}{\zeta^{k_{j}}-1} .
$$

The Eichler trace formula is closely related to the Lefschetz fixed-point formula. From (1), we can derive

$$
t=2-\chi-\bar{\chi}
$$

This is the Lefschetz fixed-point formula for closed Riemann surfaces.

Since the trace $\chi$ is invariant under base changes, it is characterized by an automorphism. Conversely, it is known that an automorphism is characterized by the trace of induced action on the space of holomorphic 1-forms. A number of articles studying the traces of automorphisms of Riemann surfaces have appeared. We refer the reader to [1] and references therein for further discussion of this point.

Fuertes and González-Diez (see [4]) applied the Lefschetz fixed-point formula to study the number of coincidences, that is, the number of points $p \in X$ with $f_{1}(p)=f_{2}(p)$ for two distinct morphisms $f_{i}: X \rightarrow Y(i=1,2)$ between Riemann surfaces. They gave a sharp bound for the number of coincidences of two morphisms.

https://doi.org/10.5186/aasfm.2017.4224

2010 Mathematics Subject Classification: Primary 30F30; Secondary 14F25.

Key words: Riemann surfaces, Eichler trace formula, Lefschetz trace formula. 
Theorem 1.1. (Fuertes and González-Diez) Let $f_{i}: X \rightarrow Y$ be two distinct morphisms of degree $d_{i}(i=1,2)$ between closed Riemann surfaces of genera $g$ and $\gamma$, respectively, and let $L\left(f_{1}, f_{2}\right)$ denote the number of coincidences appropriately counted. We have

i) $L\left(f_{1}, f_{2}\right) \leq d_{1}+2 \gamma \sqrt{d_{1} d_{2}}+d_{2}$.

ii) In case $\gamma \geq 2$, this bound is attained if and only if $Y$ is hyperelliptic and $f_{2}=J \circ f_{1}$, where $J$ denotes the hyperelliptic involution of $Y$.

Their results generalize the well known fact about the number of fixed points of automorphisms, namely, the number of fixed points is less than or equal to $2 g+2$ for an arbitrary automorphism of a closed Riemann surface. More recently, Fuertes [3] showed several results concerning the number of coincidences by composing morphisms with meromorphic functions on the target $Y$.

In [7] the author studied the case where there exists no coincidence of two distinct morphisms $f_{i}: X \rightarrow Y(i=1,2)$ between closed Riemann surfaces, namely, the case where $L\left(f_{1}, f_{2}\right)=0$. Roughly speaking, trace $\left(\left.f_{1}^{*} \circ f_{2 *}\right|_{H_{D R}^{1}(X)}\right)$ defines an innerproduct on the space of morphisms from $X$ to $Y$, where $f_{*}: H_{D R}^{1}(X) \rightarrow H_{D R}^{1}(Y)$ is defined by the property $\int_{Y} f_{*} v \wedge w=\int_{X} v \wedge f^{*} w$, for any $w \in H_{D R}^{1}(Y)$. A necessary and sufficient condition for $L\left(f_{1}, f_{2}\right)=0$ in terms of the inner-product was given. The holomorphic Lefschetz number for coincidences $L\left(f_{1}, f_{2}, \mathcal{O}\right)$ was defined as

$$
L\left(f_{1}, f_{2}, \mathcal{O}\right)=\left.\sum_{q=0}^{1}(-1)^{q} \operatorname{trace} f_{1}^{*} \circ f_{2 *}\right|_{H_{\bar{\partial}}^{0, q}(X)},
$$

to be used for the proofs.

In this paper, we investigate the holomorphic Lefschetz number more, and show that the number can be evaluated in terms of the local behavior of two morphisms around the coincidences. As a result, a generalization of the Eichler trace formula for morphisms between Riemann surfaces is given. This is all done in section 4 . We exhibit examples for the generalized Eichler trace formula in section 5. In section 6, we examine the possibility of diagonalizing matrix representations of $f_{1}^{*} \circ f_{2 *}$.

\section{The Lefschetz trace formula}

In the following, we use the notation of [4] and Chapter 3.4 of [5]. Let $X$ be a Riemann surface and let $T \in \operatorname{Aut}(X)$. We put $\Gamma_{T}=\{(p, T(p))\} \subset X \times X$ the graph of $T$. A fixed point of $T$ is corresponds to a point of intersection of the graph $\Gamma_{T}$ and the diagonal submanifold $\Delta \subset X \times X$. The Lefschetz number of $T$ is defined to be

$$
L(T)=\sharp\left(\Delta \cdot \Gamma_{T}\right) .
$$

By using integral,

$$
L(T)=\int_{\Gamma_{T}} \varphi_{\Delta}=\int_{X}(i d . \times T)^{*} \varphi_{\Delta},
$$

where $\varphi_{\Delta} \in H_{D R}^{2}(X \times X)$ is a closed form representing the cohomology class Poincaré dual to the class of $\Delta$. For each $q$ let

$$
\left\{\psi_{q, \mu}\right\}
$$

be a collection of closed $q$-forms representing a basis for $H_{\mathrm{DR}}^{q}(X)$, and let

$$
\left\{\psi_{2-q, \mu}^{*}\right\}
$$


be closed forms representing the dual basis for $H_{\mathrm{DR}}^{2-q}(X)$, i.e., such that

$$
\int_{X} \psi_{q, \mu} \wedge \psi_{2-q, \nu}^{*}=\delta_{\mu, \nu}
$$

Let $\pi_{1}$ and $\pi_{2}$ denote the two projection maps $X \times X \rightarrow X$. Then one has

$$
\varphi_{\Delta}=\sum_{q}(-1)^{q} \sum_{\mu} \pi_{1}^{*} \psi_{q, \mu} \wedge \pi_{2}^{*} \psi_{2-q, \mu}^{*}
$$

Thus we can evaluate the Lefschetz number by

$$
\begin{aligned}
L(T) & =\int_{\Gamma_{T}} \varphi_{\Delta}=\int_{X}(i d . \times T)^{*} \varphi_{\Delta}=\sum_{q}(-1)^{q} \sum_{\mu} \int_{X} \psi_{q, \mu} \wedge T^{*} \psi_{2-q, \mu}^{*} \\
& =\sum_{k=0}^{k=2}(-1)^{k} \operatorname{trace}\left(\left.T^{*}\right|_{H_{D R}^{k}(X)}\right),
\end{aligned}
$$

where $k=2-q$. The obtained formula

$$
L(T)=\sum_{k=0}^{k=2}(-1)^{k} \operatorname{trace}\left(\left.T^{*}\right|_{H_{D R}^{k}(X)}\right)
$$

is so called the Lefschetz trace formula (for two-dimensional case).

In the rest of this paper, $f_{i}: X \rightarrow Y$ will always mean two distinct morphisms of degree $d_{i}(i=1,2)$ between closed Riemann surfaces of genera $g$ and $\gamma$, respectively. For two distinct morphisms $f_{i}(i=1,2)$, we define the Lefschetz number as follows.

Definition 2.1. The Lefschetz number of two distinct morphisms $f_{i}: X \rightarrow$ $Y(i=1,2)$ is defined to be

$$
L\left(f_{1}, f_{2}\right)=\int_{X}\left(f_{1} \times f_{2}\right)^{*} \varphi_{\Delta}
$$

where $\varphi_{\Delta} \in H_{D R}^{2}(Y \times Y)$ is the Poincaré dual of the diagonal $\Delta \subset Y \times Y$.

Thus letting $\Gamma_{f_{1}, f_{2}}=\left\{p \in X \mid\left(f_{1}(p), f_{2}(p)\right)\right\} \in \mathrm{H}_{2}(Y \times Y, \mathbf{Z})$ be the homology class of the image of $X$ via $f_{1}$ and $f_{2}$, we have

$$
L\left(f_{1}, f_{2}\right)=\int_{X}\left(f_{1} \times f_{2}\right)^{*} \varphi_{\Delta}=\int_{\Gamma_{f_{1}, f_{2}}} \varphi_{\Delta}=\sharp\left(\Delta \cdot \Gamma_{f_{1}, f_{2}}\right) .
$$

Definition 2.2. Let $f: X \rightarrow Y$ be a morphism between Riemann surfaces. We define a linear map

$$
f_{*}: H_{D R}^{k}(X) \rightarrow H_{D R}^{k}(Y)
$$

by the property

$$
\int_{Y} f_{*} v \wedge w=\int_{X} v \wedge f^{*} w
$$

for any $w \in H_{D R}^{2-k}(Y)$. 
Then the analogue to (2) takes the form

$$
\begin{aligned}
L\left(f_{1}, f_{2}\right) & =\int_{X}\left(f_{1} \times f_{2}\right)^{*} \varphi_{\Delta}=\sum_{q}(-1)^{q} \sum_{\mu} \int_{X} f_{1}^{*} \psi_{q, \mu} \wedge f_{2}^{*} \psi_{2-q, \mu}^{*} \\
& =\sum_{q}(-1)^{q} \sum_{\mu} \int_{Y} f_{2 *} \circ f_{1}^{*} \psi_{q, \mu} \wedge \psi_{2-q, \mu}^{*} \\
& =\sum_{q=0}^{q=2}(-1)^{q} \operatorname{trace}\left(\left.f_{2 *} \circ f_{1}^{*}\right|_{H_{D R}^{q}(Y)}\right)=\sum_{k=0}^{k=2}(-1)^{k} \operatorname{trace}\left(\left.f_{1}^{*} \circ f_{2 *}\right|_{H_{D R}^{k}(X)}\right),
\end{aligned}
$$

where we use the same symbol $\left\{\psi_{q, \mu}\right\}$ and $\left\{\psi_{2-q, \mu}^{*}\right\}$ for the basis for $H_{\mathrm{DR}}^{q}(Y)$ and for the dual basis for $H_{\mathrm{DR}}^{2-q}(Y)$, respectively. The last equality comes from the fact that for any two matrices $A$ and $B$, the trace of $A B$ and $B A$ agree whenever the two products make sense.

Observing (3), we easily have

$$
L\left(f_{1}, f_{2}\right)=L\left(f_{2}, f_{1}\right) .
$$

Fuertes and González-Diez [4] showed the following lemma.

Lemma 2.3. $\quad$ i) $f_{1}^{*} \circ f_{2 *}: H^{0}(X) \rightarrow H^{0}(X)$ is multiplication by $d_{2}$.

ii) $f_{1}^{*} \circ f_{2 *}: H^{2}(X) \rightarrow H^{2}(X)$ is multiplication by $d_{1}$.

Thus the Lefschetz trace formula is written as

$$
L\left(f_{1}, f_{2}\right)=\sum_{k=0}^{k=2}(-1)^{k} \operatorname{trace}\left(\left.f_{1}^{*} \circ f_{2 *}\right|_{H_{D R}^{k}(X)}\right)=d_{1}-\left.\operatorname{trace} f_{1}^{*} \circ f_{2 *}\right|_{H_{D R}^{1}(X)}+d_{2} \text {. }
$$

Definition 2.4. Let $p \in X$ be a coincidence of $f_{1}$ and $f_{2}$, and let

$$
f_{1}(z)-f_{2}(z)=c_{k} z^{k}+c_{k+1} z^{k+1}+\ldots, \quad c_{k} \neq 0
$$

be the Taylor expansion of $f_{1}-f_{2}$ with respect to small parametric discs $D$ around $p$ and $D^{\prime}$ around $f_{i}(p)$. We define the multiplicity of $f_{1}$ and $f_{2}$ at $p$ to be

$$
m_{p}\left(f_{1}, f_{2}\right)=k .
$$

By the definition, $m_{p}\left(f_{1}, f_{2}\right)$ is always positive. Furthermore, one can show that (see [4])

$$
L\left(f_{1}, f_{2}\right)=\sum_{\left\{p \in X ; f_{1}(p)=f_{2}(p)\right\}} m_{p}\left(f_{1}, f_{2}\right) .
$$

Thus $L\left(f_{1}, f_{2}\right)$ is always greater than or equal to the actual number of coincidences.

\section{The holomorphic Lefschetz number}

A morphism $f: X \rightarrow Y$ acts not only on the de Rham cohomology groups but on the Dolbeault cohomology groups. Let $M$ be a compact Kähler manifold. By the Hodge decomposition,

$$
\begin{aligned}
H^{r}(M, \mathbf{C}) & \cong \bigoplus_{p+q=r} H_{\bar{\partial}}^{p, q}(M), \\
H_{\bar{\partial}}^{p, q}(M) & =\overline{H_{\bar{\partial}}^{q, p}(M)} .
\end{aligned}
$$

Thus, for a Riemann surface $X$,

$$
H^{1}(X, \mathbf{C}) \cong H_{\bar{\partial}}^{1,0}(X) \bigoplus H_{\bar{\partial}}^{0,1}(X)
$$


holds, where we may identify $H_{\bar{\partial}}^{1,0}(X)$ with the space of holomorphic 1-forms and $H_{\bar{\partial}}^{0,1}(X)$ being the complex conjugate of $H_{\bar{\partial}}^{1,0}(X) . H^{0}$ and $H^{2}$ are trivial in this case. Now the Lefschetz number $L\left(f_{1}, f_{2}\right)$ is written as

$$
L\left(f_{1}, f_{2}\right)=\int_{X}\left(f_{1} \times f_{2}\right)^{*} \varphi_{\Delta}=\sum_{p, q}(-1)^{p+q} \operatorname{trace}\left(\left.f_{1}^{*} \circ f_{2 *}\right|_{H_{\frac{p}{\partial}}^{p, q}(X)}\right) .
$$

Let $\pi_{1}$ and $\pi_{2}$ denote the two projection maps $Y \times Y \rightarrow Y$. For each $p$ and $q$ let

$$
\left\{\psi_{p, q, \mu}\right\}
$$

be a collection of $\bar{\partial}$-closed $(p, q)$-forms representing a basis for $H_{\bar{\partial}}^{p, q}(Y)$, and let

$$
\left\{\psi_{1-p, 1-q, \mu}^{*}\right\}
$$

be $\bar{\partial}$-closed forms representing the dual basis for $H_{\bar{\partial}}^{1-p, 1-q}(Y)$ under the pairing

$$
H_{\bar{\partial}}^{p, q}(Y) \otimes H_{\bar{\partial}}^{1-p, 1-q}(Y) \rightarrow \mathbf{C}
$$

given by

$$
\psi \otimes \varphi \mapsto \int_{Y} \psi \wedge \varphi
$$

A basis for $H_{\bar{\partial}}^{1,1}(Y \times Y)$ is represented by the forms

$$
\left\{\varphi_{p, q, \mu, \nu}=\pi_{1}^{*} \psi_{p, q, \mu} \wedge \pi_{2}^{*} \psi_{1-p, 1-q, \nu}^{*}\right\}
$$

and the dual basis for $H_{\bar{\partial}}^{1,1}(Y \times Y)$ is represented by

$$
\left\{\varphi_{1-p, 1-q, \mu, \nu}^{*}=\pi_{1}^{*} \psi_{1-p, 1-q, \mu}^{*} \wedge \pi_{2}^{*} \psi_{p, q, \nu}\right\} .
$$

The Dolbeault class of the diagonal is represented by the form

$$
\varphi_{\Delta}=\sum_{p, q, \mu}(-1)^{p+q} \varphi_{p, q, \mu, \mu} .
$$

Set

$$
\varphi_{\Delta}^{0}=\sum_{q, \mu}(-1)^{q} \varphi_{0, q, \mu, \mu}=\varphi_{0,0}-\sum_{\mu} \varphi_{0,1, \mu, \mu} .
$$

We put

$$
\eta_{\Delta}^{0}\left(\Gamma_{f_{1}, f_{2}}\right)=\int_{\Gamma_{f_{1}, f_{2}}} \varphi_{\Delta}^{0}
$$

integration of $\varphi_{\Delta}^{0}$ over $\Gamma_{f_{1}, f_{2}}$. We have

$$
\begin{aligned}
\eta_{\Delta}^{0}\left(\Gamma_{f_{1}, f_{2}}\right) & =\int_{\Gamma_{f_{1}, f_{2}}} \varphi_{\Delta}^{0}=\int_{X}\left(f_{1} \times f_{2}\right)^{*} \varphi_{\Delta}^{0}=\int_{X} \sum_{q, \mu}(-1)^{q} f_{1}^{*} \psi_{0, q, \mu} \wedge f_{2}^{*} \psi_{1,1-q, \mu}^{*} \\
& =\int_{Y} \sum_{q, \mu}(-1)^{q} f_{2 *} \circ f_{1}^{*} \psi_{0, q, \mu} \wedge \psi_{1,1-q, \mu}^{*}=\left.\sum_{q=0}^{1}(-1)^{q} \operatorname{trace} f_{2 *} \circ f_{1}^{*}\right|_{H_{\frac{\partial}{\partial}}^{0, q}(Y)} \\
& =\left.\sum_{q=0}^{1}(-1)^{q} \operatorname{trace} f_{1}^{*} \circ f_{2 *}\right|_{H_{\frac{\partial}{\partial}}^{0, q}(X)} .
\end{aligned}
$$

The last equality comes from the fact that for any two matrices $A$ and $B$, the trace of $A B$ and $B A$ agree whenever the two products make sense. 
Definition 3.1. We define the holomorphic Lefschetz number of $\left(f_{1}, f_{2}\right)$ to be

$$
L\left(f_{1}, f_{2}, \mathcal{O}\right)=\left.\sum_{q=0}^{1}(-1)^{q} \operatorname{trace} f_{1}^{*} \circ f_{2 *}\right|_{H_{\bar{\partial}}^{0, q}(X)} .
$$

By Lemma 2.3 i), we see

$$
L\left(f_{1}, f_{2}, \mathcal{O}\right)=d_{2}-\left.\operatorname{trace} f_{1}^{*} \circ f_{2 *}\right|_{H_{\frac{\partial}{O}}^{0,1}(X)} .
$$

We also have

$$
L\left(f_{2}, f_{1}, \mathcal{O}\right)=d_{1}-\left.\operatorname{trace} f_{1}^{*} \circ f_{2 *}\right|_{H_{\bar{\partial}}^{1,0}(X)}
$$

since

$$
\begin{aligned}
\int_{X}\left(f_{2} \times f_{1}\right)^{*} \varphi_{\Delta}^{0} & =\int_{X} \sum_{q, \mu}(-1)^{q} f_{2}^{*} \psi_{0, q, \mu} \wedge f_{1}^{*} \psi_{1,1-q, \mu}^{*} \\
& =\int_{Y} \sum_{q, \mu}(-1)^{q} \psi_{0, q, \mu} \wedge f_{2 *} \circ f_{1}^{*} \psi_{1,1-q, \mu}^{*} \\
& =\left.\sum_{q=0}^{1}(-1)^{1-q} \operatorname{trace} f_{2 *} \circ f_{1}^{*}\right|_{H_{\frac{\partial}{\partial}}^{1, q}(Y)} \\
& =\left.\sum_{q=0}^{1}(-1)^{1-q} \operatorname{trace} f_{1}^{*} \circ f_{2 *}\right|_{H_{\frac{\partial}{\partial}}^{1, q}(X)} .
\end{aligned}
$$

Summing (6) and (7), we have

$$
L\left(f_{1}, f_{2}\right)=L\left(f_{1}, f_{2}, \mathcal{O}\right)+L\left(f_{2}, f_{1}, \mathcal{O}\right) .
$$

In [7], the holomorphic Lefschetz number was used to show a theorem giving a condition for $L\left(f_{1}, f_{2}\right)=0$. As a consequence of the theorem, the following corollary was deduced.

Corollary 3.2. Let $f_{i}: X \rightarrow Y$ be two distinct morphisms of degree $d_{i}(i=1,2)$ between closed Riemann surfaces of genera $g$ and $\gamma=1$, respectively. The following two conditions are equivalent.

1) $L\left(f_{1}, f_{2}\right)=0$.

2) The difference between $f_{1}$ and $f_{2}$ is only a translation on the torus $Y$.

For details, we refer to [7].

\section{The Eichler trace formula}

According to the method in [5], we can evaluate the number $\eta_{\Delta}^{0}\left(\Gamma_{f_{1}, f_{2}}\right)$ in terms of the local behavior of $f_{1}$ and $f_{2}$ around their coincidences.

Let $A^{p, q}(Y)$ denote the space of differential forms of type $(p, q)$, and let

$$
A^{\left(p_{1}, q_{1}\right),\left(p_{2}, q_{2}\right)}(Y \times Y)
$$

denote the space of differential forms of bitype $\left(p_{1}, q_{1}\right),\left(p_{2}, q_{2}\right)$ where $\left(p_{1}, q_{1}\right)$ and $\left(p_{2}, q_{2}\right)$ come from the first and the second factor of the product $Y \times Y$, respectively. We have the decomposition of forms on $Y \times Y$ into bitype

$$
A^{p, q}(Y \times Y)=\bigoplus_{\substack{p_{1}+p_{2}=p \\ q_{1}+q_{2}=q}} A^{\left(p_{1}, q_{1}\right),\left(p_{2}, q_{2}\right)}(Y \times Y) .
$$


We denote by $T_{\Delta}$ the current of the diagonal $\Delta \subset Y \times Y$. Let $T_{\Delta}^{0}$ be the component of $T_{\Delta}$ of bitype $(0, *),(1,1-*)$, where $*=0,1$, that is, the current defined by the linear function

$$
T_{\Delta}^{0}(\psi)=\int_{\Delta} \sum_{q=0}^{1} \psi^{(1,1-q),(0, q)}
$$

on test forms $\psi$. Then $T_{\Delta}^{0}$ is $\bar{\partial}$-closed and represents the Dolbeault cohomology class $\eta_{\Delta}^{0}$. We will smooth the current, namely, solve the equation of currents

$$
T_{\Delta}^{0}=\varphi+\bar{\partial} k
$$

with $k$ any $(1,0)$-current and $\varphi$ a smooth form. Then we will have

$$
\eta_{\Delta}^{0}\left(\Gamma_{f_{1}, f_{2}}\right)=\int_{\Gamma_{f_{1}, f_{2}}} \varphi
$$

The Bochner-Martinelli kernel on $\mathbf{C} \times \mathbf{C}$ is given by

$$
k(z, \zeta)=C_{1} \frac{d \zeta}{z-\zeta}
$$

where

$$
C_{1}=\frac{-1}{2 \pi i}
$$

Apparently, $\bar{\partial} k(z, \zeta)=0$ on $\mathbf{C} \times \mathbf{C}-\Delta$ and therefore the current defined by $k(z, \zeta)$ has distributional derivative $\bar{\partial} k$ supported on $\Delta$. Further, it is known that $\bar{\partial} k=T_{\Delta}^{0}$ in the sense of distributions. This gives the smoothing of $T_{\Delta}^{0}$ in $\mathbf{C} \times \mathbf{C}$.

Let $f_{i}: X \rightarrow Y$ be two distinct morphisms of degree $d_{i}(i=1,2)$ between closed Riemann surfaces and let $\left\{p_{\alpha}\right\}$ be their coincidences possibly empty set. Put the image of coincidences $q_{\alpha}=f_{i}\left(p_{\alpha}\right)$. We denote by $B_{\epsilon}\left(q_{\alpha}, q_{\alpha}\right)$ the ball of radius $\epsilon$ around $\left(q_{\alpha}, q_{\alpha}\right)$ in $Y \times Y$, and let $\rho_{\alpha}$ be a bump function with $\rho_{\alpha} \equiv 1$ in $B_{\epsilon}\left(q_{\alpha}, q_{\alpha}\right)$ and $\rho_{\alpha} \equiv 0$ in $Y \times Y-B_{2 \epsilon}\left(q_{\alpha}, q_{\alpha}\right)$. Let $k_{\epsilon}$ be the current on $Y \times Y$ given by

$$
k_{\epsilon}=\sum_{\alpha} \rho_{\alpha} \cdot k\left(z_{\alpha}, \zeta_{\alpha}\right)
$$

where $k\left(z_{\alpha}, \zeta_{\alpha}\right)$ is the Bochner-Martinelli kernel.

In $B_{\epsilon}\left(q_{\alpha}, q_{\alpha}\right)$

and if we put

$$
\bar{\partial} k_{\epsilon}=\bar{\partial} k\left(z_{\alpha}, \zeta_{\alpha}\right)=T_{\Delta}^{0}
$$

$$
\varphi=T_{\Delta}^{0}-\bar{\partial} k_{\epsilon}
$$

$\varphi$ is a $\bar{\partial}$-closed current representing $\eta_{\Delta}^{0}$, smooth in an open set containing $\Gamma_{f_{1}, f_{2}}$ and equal to $-\bar{\partial} k_{\epsilon}$ away from $\Delta$. Let $\xi_{\alpha}$ be a local coordinate around $p_{\alpha}$. We evaluate

$$
\begin{aligned}
\eta_{\Delta}^{0}\left(\Gamma_{f_{1}, f_{2}}\right) & =\int_{\Gamma_{f_{1}, f_{2}}} \varphi=-\int_{\Gamma_{f_{1}, f_{2}}-\cup B_{\epsilon}\left(q_{\alpha}, q_{\alpha}\right)} \bar{\partial} k_{\epsilon}=\sum_{\alpha} \int_{\partial\left(\Gamma_{\left.f_{1}, f_{2} \cap B_{\epsilon}\left(q_{\alpha}, q_{\alpha}\right)\right)} k_{\epsilon}\right.} k\left(f_{1}\left(\xi_{\alpha}\right), f_{2}\left(\xi_{\alpha}\right)\right)=\sum_{\alpha} C_{1} \int_{\left|\xi_{\alpha}\right|=\epsilon} \frac{f_{2}^{\prime}\left(\xi_{\alpha}\right)}{f_{1}\left(\xi_{\alpha}\right)-f_{2}\left(\xi_{\alpha}\right)} d \xi_{\alpha} .
\end{aligned}
$$

If $\left\{p_{\alpha}\right\}$ is the empty set, it is easy to see that the third integral is 0 . Then

$$
C_{1} \int_{\left|\xi_{\alpha}\right|=\epsilon} \frac{f_{2}^{\prime}\left(\xi_{\alpha}\right)}{f_{1}\left(\xi_{\alpha}\right)-f_{2}\left(\xi_{\alpha}\right)} d \xi_{\alpha}=\operatorname{Res}\left(\frac{f_{2}^{\prime}\left(\xi_{\alpha}\right)}{f_{2}\left(\xi_{\alpha}\right)-f_{1}\left(\xi_{\alpha}\right)} ; p_{\alpha}\right),
$$


where

$$
\operatorname{Res}\left(\frac{f_{2}^{\prime}\left(\xi_{\alpha}\right)}{f_{2}\left(\xi_{\alpha}\right)-f_{1}\left(\xi_{\alpha}\right)} ; p_{\alpha}\right)
$$

denotes the residue of the function at $p_{\alpha}$, which is independent of the choice of $\epsilon$. Thus

$$
\eta_{\Delta}^{0}\left(\Gamma_{f_{1}, f_{2}}\right)=\sum_{\alpha} \operatorname{Res}\left(\frac{f_{2}^{\prime}\left(\xi_{\alpha}\right)}{f_{2}\left(\xi_{\alpha}\right)-f_{1}\left(\xi_{\alpha}\right)} ; p_{\alpha}\right),
$$

and we have established

Theorem 4.1. Let $f_{i}: X \rightarrow Y$ be two distinct morphisms of degree $d_{i}(i=1,2)$ between closed Riemann surfaces and let $\left\{p_{\alpha}\right\}$ be their coincidences possibly empty set. The holomorphic Lefschetz number of $\left(f_{1}, f_{2}\right)$ is given by

$$
L\left(f_{1}, f_{2}, \mathcal{O}\right)=\sum_{\alpha} \operatorname{Res}\left(\frac{f_{2}^{\prime}\left(\xi_{\alpha}\right)}{f_{2}\left(\xi_{\alpha}\right)-f_{1}\left(\xi_{\alpha}\right)} ; p_{\alpha}\right) .
$$

If $\left\{p_{\alpha}\right\}$ is empty, then we take the right hand side to be 0 .

By the definition of $L\left(f_{1}, f_{2}, \mathcal{O}\right)$ and the fact that

$$
\left.\operatorname{trace} f_{1}^{*} \circ f_{2 *}\right|_{H_{\bar{\partial}}^{0,1}(X)}=\overline{\left.\operatorname{trace} f_{1}^{*} \circ f_{2 *}\right|_{H_{\bar{\partial}}^{1,0}(X)}},
$$

we easily derive

Corollary 4.2. (The Eichler trace formula for coincidences) With the same notation as in Theorem 4.1, we have

$$
\left.\operatorname{trace} f_{1}^{*} \circ f_{2 *}\right|_{H_{\bar{\partial}}^{1,0}(X)}=d_{2}-\sum_{\alpha} \overline{\operatorname{Res}\left(\frac{f_{2}^{\prime}\left(\xi_{\alpha}\right)}{f_{2}\left(\xi_{\alpha}\right)-f_{1}\left(\xi_{\alpha}\right)} ; p_{\alpha}\right)} .
$$

For automorphisms, namely if $X=Y$ and $f_{2}$ is the identity map on $X$ and $f_{1} \neq \mathrm{id}$. then a coincidence is a fixed point of $f_{1}$ and

$$
\operatorname{Res}\left(\frac{f_{2}^{\prime}\left(\xi_{\alpha}\right)}{f_{2}\left(\xi_{\alpha}\right)-f_{1}\left(\xi_{\alpha}\right)} ; p_{\alpha}\right)=\frac{1}{1-f_{1}^{\prime}\left(\xi_{\alpha}\left(p_{\alpha}\right)\right)} .
$$

Substituting this into Corollary 4.2, we obtain the Eichler trace formula

$$
\left.\operatorname{trace} f_{1}^{*}\right|_{H_{\frac{\partial}{\partial}}^{1,0}(X)}=1-\sum_{\alpha} \frac{1}{1-f_{1}^{\prime}\left(\xi_{\alpha}\left(p_{\alpha}\right)\right)}=1-\sum_{\alpha} \frac{f_{1}^{\prime}\left(\xi_{\alpha}\left(p_{\alpha}\right)\right)}{f_{1}^{\prime}\left(\xi_{\alpha}\left(p_{\alpha}\right)\right)-1},
$$

since $\left|f_{1}^{\prime}\left(\xi_{\alpha}\left(p_{\alpha}\right)\right)\right|=1$.

Remark. For another proof of the Eichler trace formula, we refer to [2] in which for an automorphism $T$ of a Riemann surface $X, \operatorname{tr} T$ denotes trace $\left.\left(T^{-1}\right)^{*}\right|_{H_{\frac{\partial}{\partial}}^{1,0}(X)}$ in our notation. Also in [2], the genera of Riemann surfaces are assumed to be $>1$ although we do not exclude tori.

We return to Theorem 4.1. Changing the order of $f_{1}$ and $f_{2}$, we have

$$
L\left(f_{2}, f_{1}, \mathcal{O}\right)=\sum_{\alpha} \operatorname{Res}\left(\frac{f_{1}^{\prime}\left(\xi_{\alpha}\right)}{f_{1}\left(\xi_{\alpha}\right)-f_{2}\left(\xi_{\alpha}\right)} ; p_{\alpha}\right) .
$$


Thus

$$
\begin{aligned}
& L\left(f_{1}, f_{2}, \mathcal{O}\right)+L\left(f_{2}, f_{1}, \mathcal{O}\right) \\
& =\sum_{\alpha} \operatorname{Res}\left(\frac{f_{2}^{\prime}\left(\xi_{\alpha}\right)}{f_{2}\left(\xi_{\alpha}\right)-f_{1}\left(\xi_{\alpha}\right)} ; p_{\alpha}\right)+\sum_{\alpha} \operatorname{Res}\left(\frac{f_{1}^{\prime}\left(\xi_{\alpha}\right)}{f_{1}\left(\xi_{\alpha}\right)-f_{2}\left(\xi_{\alpha}\right)} ; p_{\alpha}\right) \\
& =\sum_{\alpha} \operatorname{Res}\left(\frac{f_{2}^{\prime}\left(\xi_{\alpha}\right)-f_{1}^{\prime}\left(\xi_{\alpha}\right)}{f_{2}\left(\xi_{\alpha}\right)-f_{1}\left(\xi_{\alpha}\right)} ; p_{\alpha}\right) .
\end{aligned}
$$

Recalling (5), we have again obtained

$$
L\left(f_{1}, f_{2}\right)=L\left(f_{1}, f_{2}, \mathcal{O}\right)+L\left(f_{2}, f_{1}, \mathcal{O}\right) .
$$

If the multiplicity of $f_{1}$ and $f_{2}$ at $p_{\alpha}$ is 1 , then

$$
\operatorname{Res}\left(\frac{f_{2}^{\prime}\left(\xi_{\alpha}\right)}{f_{2}\left(\xi_{\alpha}\right)-f_{1}\left(\xi_{\alpha}\right)} ; p_{\alpha}\right)=\frac{f_{2}^{\prime}\left(\xi_{\alpha}\left(p_{\alpha}\right)\right)}{f_{2}^{\prime}\left(\xi_{\alpha}\left(p_{\alpha}\right)\right)-f_{1}^{\prime}\left(\xi_{\alpha}\left(p_{\alpha}\right)\right)} .
$$

In this case, we have

Corollary 4.3. Let the conditions of Theorem 4.1 hold, and suppose that every coincidence has multiplicity one. Then the difference in degree between $f_{1}$ and $f_{2}$ is given by

$$
d_{2}-d_{1}=\sum_{\alpha} \frac{\left|f_{2}^{\prime}\left(\xi_{\alpha}\left(p_{\alpha}\right)\right)\right|^{2}-\left|f_{1}^{\prime}\left(\xi_{\alpha}\left(p_{\alpha}\right)\right)\right|^{2}}{\left|f_{2}^{\prime}\left(\xi_{\alpha}\left(p_{\alpha}\right)\right)-f_{1}^{\prime}\left(\xi_{\alpha}\left(p_{\alpha}\right)\right)\right|^{2}} .
$$

Proof. Recalling that

$$
\begin{aligned}
& L\left(f_{1}, f_{2}, \mathcal{O}\right)=d_{2}-\left.\operatorname{trace} f_{1}^{*} \circ f_{2 *}\right|_{H_{\bar{\partial}}^{0,1}(X)}, \\
& L\left(f_{2}, f_{1}, \mathcal{O}\right)=d_{1}-\left.\operatorname{trace} f_{1}^{*} \circ f_{2 *}\right|_{H_{\bar{\partial}}^{1,0}(X)},
\end{aligned}
$$

and

$$
\left.\operatorname{trace} f_{1}^{*} \circ f_{2 *}\right|_{H_{\bar{\partial}}^{0,1}(X)}=\overline{\left.\operatorname{trace} f_{1}^{*} \circ f_{2 *}\right|_{H_{\bar{\partial}}^{1,0}(X)}},
$$

we easily derive the conclusion from Theorem 4.1 .

\section{Examples}

In this section, we exhibit examples for Corollary 4.2 .

Example 1. Consider the torus

$$
T=\mathbf{C} /(i, 1) \mathbf{Z}^{2} .
$$

For any positive integer $n$, define the multiplication by $\mathrm{n}$ on $T$

$$
n_{T}: T \rightarrow T
$$

by

$$
x \mapsto n x .
$$

Let $n_{1}$ and $n_{2}$ be positive integers distinct from each other. We consider the coincidences of $f_{1}=n_{1 T}$ and $f_{2}=n_{2 T}$. The number of coincidences is $\left(n_{1}-n_{2}\right)^{2}$. Now, we recall that the push-forward of a holomorphic 1-form $f_{*} \omega$ is expressed by the local behavior of the morphism $f$ and the local value of the form $\omega$ as below (we refer to $[3$, p. 344]). Let $f: X \rightarrow Y$ be a morphism between Riemann surfaces of degree $d$ and $\omega$ be a holomorphic 1-form on $X$. Let $U^{\prime}$ be an open set of $Y$ with the property that 
$f^{-1}\left(U^{\prime}\right)$ is the disjoint union of open sets $U_{i}(i=1,2, \cdots, d)$ such that the restriction of $f$ to each of them is an isomorphism. We assign to each such open set $U^{\prime}$ the form

$$
\left.\omega^{\prime}\right|_{U^{\prime}}=\sum_{i=1}^{d}\left(\left(\left.f\right|_{U_{i}}\right)^{-1}\right)^{*} \omega
$$

Then we can obtain a globally well defined holomorphic form $\omega^{\prime}$ and this is $f_{*} \omega$.

Observing this expression, we calculate the left hand side of the equation in Corollary 4.2 to be $n_{1} n_{2}$. The right hand side is $n_{2}^{2}-\left(n_{1}-n_{2}\right)^{2} \cdot \frac{n_{2}}{n_{2}-n_{1}}$ which is equal to $n_{1} n_{2}$.

Fuertes [3] computed the multiplicities of coincidences of morphisms given below.

Example 2. Let $X$ and $Y$ be hyperelliptic Riemann surfaces defined by the equations $y^{2}=x^{2 n}-1$ and $y^{2}=x^{n}-1$ with $n$ even, respectively. Then $X$ is of genus $n-1$ and $Y$ is of genus $(n-2) / 2$. Let $f_{j}: X \rightarrow Y(0 \leq j \leq n-1)$ be morphisms defined by

$$
f_{j}(x, y)=\left(\zeta^{j} x^{2}, y\right)
$$

where $\zeta=e^{2 \pi i / n}$. Then $\operatorname{deg} f_{j}=2$ for any $j$. We put

$$
p_{1}=(x, y)=(0,+i), \quad p_{2}=(x, y)=(0,-i)
$$

on $X$. Then $p_{1}$ and $p_{2}$ are coincidences of $f_{0}$ and $f_{k}$ for any $k \neq 0$. It is seen that the multiplicity of each of these coincidences is 2 as indicated in [3]. Taking the variable $x$ of defining equation for $X$ as a local coordinate around $p_{i}(i=1,2)$, we compute

$$
\operatorname{Res}\left(\frac{f_{0}^{\prime}(x)}{f_{0}(x)-f_{k}(x)} ; p_{i}\right)=\frac{1}{2 \pi i} \int_{|x|=\epsilon} \frac{2 x}{x^{2}-\zeta^{k} x^{2}} d x=\frac{2}{1-\zeta^{k}} .
$$

We denote by $\infty_{1}$ and $\infty_{2}$ the two points at infinity of $X$. These points are the rest of the coincidences of $f_{0}$ and $f_{k}$. We take $s=x^{-1}$ as a local coordinate around $\infty_{1}$. Then the local expression of $f_{0}$ around $\infty_{1}$ is

$$
s \mapsto\left(\frac{1}{s}, \frac{\sqrt{1-s^{2 n}}}{s^{n}}\right) \mapsto\left(\frac{1}{s^{2}}, \frac{\sqrt{1-s^{2 n}}}{s^{n}}\right) \mapsto s^{2},
$$

and similarly the local expression of $f_{k}$ around $\infty_{1}$ is

$$
s \mapsto \frac{s^{2}}{\zeta^{k}}
$$

Thus we compute

$$
\operatorname{Res}\left(\frac{f_{0}^{\prime}(s)}{f_{0}(s)-f_{k}(s)} ; \infty_{1}\right)=\frac{1}{2 \pi i} \int_{|s|=\epsilon} \frac{2 s}{s^{2}-\zeta^{-k} s^{2}} d s=\frac{2}{1-\zeta^{-k}} .
$$

Similarly, we obtain

$$
\operatorname{Res}\left(\frac{f_{0}^{\prime}(s)}{f_{0}(s)-f_{k}(s)} ; \infty_{2}\right)=\frac{2}{1-\zeta^{-k}}
$$

It is known that

$$
\frac{d x}{y}, \frac{x d x}{y}, \cdots, \frac{x^{n-2} d x}{y}
$$

form a basis for $H_{\bar{\partial}}^{1,0}(X)$. The eigenvectors of $f_{k}^{*} \circ f_{0 *}$ are pull-back forms via $f_{0}$

$$
\frac{x d x}{y}, \frac{x^{3} d x}{y}, \cdots, \frac{x^{n-3} d x}{y}
$$


corresponding eigenvalues being

$$
2 \zeta^{2 k}, 2 \zeta^{4 k}, \cdots, 2 \zeta^{(n-2) k},
$$

where the factor 2 comes from $\operatorname{deg} f_{0}$. Then the left hand side of the equation in Corollary 4.2 is

$$
\left.\operatorname{trace} f_{k}^{*} \circ f_{0 *}\right|_{H_{\bar{\partial}}^{1,0}(X)}=\sum_{l=1}^{\frac{n-2}{2}} 2 \zeta^{2 k l}=2 \zeta^{2 k} \frac{1-\zeta^{2 k\left(\frac{n}{2}-1\right)}}{1-\zeta^{2 k}}=-2,
$$

and the right hand side is

$$
\operatorname{deg} f_{0}-\sum_{\alpha} \overline{\operatorname{Res}\left(\frac{f_{0}^{\prime}(s)}{f_{0}(s)-f_{k}(s)} ; p_{\alpha}\right)}=2-2 \overline{\frac{2}{1-\zeta^{k}}}-2 \overline{\frac{2}{1-\zeta^{-k}}}=-2 .
$$

\section{Diagonalization of $f_{1}^{*} \circ f_{2 *}$}

Our purpose in this section is to examine the possibility of diagonalizing matrix representations of $f_{1}^{*} \circ f_{2 *}$. We recall that the vector space $H_{\bar{\partial}}^{1,0}(X)$ carries a hermitian inner-product defined by

$$
\langle v, w\rangle_{X}=i \int_{X} v \wedge \bar{w}
$$

We denote by

$$
\langle v, w\rangle_{Y}
$$

the hermitian inner-product on $H_{\bar{\partial}}^{1,0}(Y)$ as well. Then for any $v \in H_{\bar{\partial}}^{1,0}(X)$ and $w \in H_{\bar{\partial}}^{1,0}(Y)$,

$$
\left\langle f_{i *} v, w\right\rangle_{Y}=i \int_{Y} f_{i *} v \wedge \bar{w}=i \int_{X} v \wedge \overline{f_{i}^{*} w}=\left\langle v, f_{i}^{*} w\right\rangle_{X}
$$

Let

$$
\Omega=\left\{\omega_{1}, \cdots, \omega_{g}\right\}
$$

be an orthonormal basis for $H_{\bar{\partial}}^{1,0}(X)$ and let

$$
\Lambda=\left\{\lambda_{1}, \cdots, \lambda_{\gamma}\right\}
$$

be an orthonormal basis for $H_{\bar{\partial}}^{1,0}(Y)$. We denote by $A$ the matrix representation of $f_{i *}$ with respect to $\Omega$ and $\Lambda$. Then (8) means that

$$
A^{*}=\overline{{ }^{t} A}
$$

is the matrix representation of $f_{i}^{*}$ with respect to $\Lambda$ and $\Omega$. This observation gives

Lemma 6.1. Let $A$ be the matrix representation of $f_{i *}: H_{\bar{\partial}}^{1,0}(X) \rightarrow H_{\bar{\partial}}^{1,0}(Y)$ with respect to orthonormal bases. Then the matrix representation of $f_{i}^{*}$ is $A^{*}$.

Now we will show

Theorem 6.2. Let $f_{i}: X \rightarrow Y(i=1,2)$ be distinct morphisms between closed Riemann surfaces of genera greater than one. Let $\Omega=\left\{\omega_{1}, \cdots, \omega_{g}\right\}$ be an orthonormal basis for $H_{\bar{\partial}}^{1,0}(X)$. A matrix representation of $f_{1}^{*} \circ f_{2 *}$ with respect to $\Omega$ is diagonalizable by a unitary matrix if and only if there is an automorphism $h$ of $Y$ with $f_{1}=h \circ f_{2}$. 
Proof. A matrix is diagonalizable by a unitary matrix if and only if it is a normal matrix. Thus we will show that there is an automorphism $h$ of $Y$ with $f_{1}=h \circ f_{2}$ if and only if the matrix representation of $f_{1}^{*} \circ f_{2 *}$ is normal.

Suppose that there is an automorphism $h$ of $Y$ with $f_{1}=h \circ f_{2}$. Let $\Lambda=$ $\left\{\lambda_{1}, \cdots, \lambda_{\gamma}\right\}$ be an orthonormal basis for $H_{\bar{\partial}}^{1,0}(Y)$. Let $\mathcal{F}_{i}(i=1,2)$ and $\mathcal{H}$ denote the matrix representations of $f_{i *}$ and $h_{*}$ respectively with respect to $\Omega$ and $\Lambda$. It suffices to show that $\mathcal{F}_{1}^{*} \mathcal{F}_{2}$ is a normal matrix. Using Lemma 6.1, we compute

$$
\left(\mathcal{F}_{1}^{*} \mathcal{F}_{2}\right)^{*}\left(\mathcal{F}_{1}^{*} \mathcal{F}_{2}\right)=\left(\mathcal{F}_{2}^{*} \mathcal{H}^{*} \mathcal{F}_{2}\right)^{*}\left(\mathcal{F}_{2}^{*} \mathcal{H}^{*} \mathcal{F}_{2}\right)=\mathcal{F}_{2}^{*} \mathcal{H} \mathcal{F}_{2} \mathcal{F}_{2}^{*} \mathcal{H}^{*} \mathcal{F}_{2}=d_{2} \mathcal{F}_{2}^{*} \mathcal{F}_{2}
$$

On the other hand,

$$
\left(\mathcal{F}_{1}^{*} \mathcal{F}_{2}\right)\left(\mathcal{F}_{1}^{*} \mathcal{F}_{2}\right)^{*}=\left(\mathcal{F}_{2}^{*} \mathcal{H}^{*} \mathcal{F}_{2}\right)\left(\mathcal{F}_{2}^{*} \mathcal{H}^{*} \mathcal{F}_{2}\right)^{*}=\mathcal{F}_{2}^{*} \mathcal{H}^{*} \mathcal{F}_{2} \mathcal{F}_{2}^{*} \mathcal{H} \mathcal{F}_{2}=d_{2} \mathcal{F}_{2}^{*} \mathcal{F}_{2}
$$

Thus we see that a matrix representation of $\mathcal{F}_{1}^{*} \mathcal{F}_{2}$ is normal.

Conversely, we assume that the matrix representation of $f_{1}^{*} \circ f_{2 *}$ is normal. Then $\left(\mathcal{F}_{1}^{*} \mathcal{F}_{2}\right)^{*}\left(\mathcal{F}_{1}^{*} \mathcal{F}_{2}\right)=\left(\mathcal{F}_{1}^{*} \mathcal{F}_{2}\right)\left(\mathcal{F}_{1}^{*} \mathcal{F}_{2}\right)^{*}$. We compute

$$
\left(\mathcal{F}_{1}^{*} \mathcal{F}_{2}\right)^{*}\left(\mathcal{F}_{1}^{*} \mathcal{F}_{2}\right)=\mathcal{F}_{2}^{*} \mathcal{F}_{1} \mathcal{F}_{1}^{*} \mathcal{F}_{2}=d_{1} \mathcal{F}_{2}^{*} \mathcal{F}_{2}
$$

and

Thus we have

$$
\left(\mathcal{F}_{1}^{*} \mathcal{F}_{2}\right)\left(\mathcal{F}_{1}^{*} \mathcal{F}_{2}\right)^{*}=\mathcal{F}_{1}^{*} \mathcal{F}_{2} \mathcal{F}_{2}^{*} \mathcal{F}_{1}=d_{2} \mathcal{F}_{1}^{*} \mathcal{F}_{1}
$$

$$
d_{1} f_{2}^{*} \circ f_{2 *}=d_{2} f_{1}^{*} \circ f_{1 *} .
$$

This means that there is an automorphism $h$ of $Y$ with $f_{1}=h \circ f_{2}$ by Kani's rigidity theorem [6, p. 186, Theorem 2].

\section{References}

[1] Breuer, T.: Characters and automorphism groups of compact Riemann surfaces. - London Math. Soc. Lecture Note Ser. 280, Cambridge Univ. Press, Cambridge, 2000.

[2] Farkas, H. M., and I. Kra: Riemann surfaces. - Springer-Verlag, New York, Heidelberg, and Berlin, 2nd edition, 1991.

[3] Fuertes, Y.: Some bounds for the number of coincidences of morphisms between closed Riemann surfaces. - Israel J. Math. 109, 1999, 1-12.

[4] Fuertes, Y., and G. GonzÁlez-Diez: On the number of coincidences of morphisms between closed Riemann surfaces. - Publ. Mat. 37, 1993, 339-353.

[5] Griffiths, P., and J. Harris: Principles of algebraic geometry. - Wiley Classics Library, John Wiley \& Sons, Inc., New York, Chichester, and Brisbane, 1994.

[6] KANi, E.: Bounds on the number of non-rational subfields of a function field. - Invent. Math. 85, 1986, 185-198.

[7] Tanabe, M.: Morphisms of closed Riemann surfaces and Lefschetz trace formula. - Proc. Amer. Math. Soc. 138:4, 2010, 1295-1303.

Received 13 February 2016 • Accepted 2 September 2016 